\title{
Design and Implementation of a Distributed Crawler and Filtering Processor *
}

\author{
Demetrios Zeinalipour-Yazti ${ }^{1}$ and Marios Dikaiakos ${ }^{2}$ \\ 1 Dept. of Computer Science and Engineering \\ University of California, Riverside CA 92507, USA, \\ csyiazti@cs.ucr.edu \\ 2 Dept. of Computer Science \\ University of Cyprus, PO Box 20537, Nicosia, Cyprus, \\ mdd@ucy.ac.cy
}

\begin{abstract}
Web crawlers are the key component of services running on Internet and providing searching and indexing support for the entire Web, for corporate Intranets and large portal sites. More recently, crawlers have also been used as tools to conduct focused Web searches and to gather data about the characteristics of the WWW. In this paper, we study the employment of crawlers as a programmable, scalable, and distributed component in future Internet middleware infrastructures and proxy services. In particular, we present the architecture and implementation of, and experimentation with WebRACE, a high-performance, distributed Web crawler, filtering server and object cache. We address the challenge of designing and implementing modular, open, distributed, and scalable crawlers, using Java. We describe our design and implementation decisions, and various optimizations. We discuss the advantages and disadvantages of using Java to implement the WebRACE-crawler, and present an evaluation of its performance. WebRACE is designed in the context of eRACE, an extensible Retrieval Annotation Caching Engine, which collects, annotates and disseminates information from heterogeneous Internet sources and protocols, according to XML-encoded user profiles that determine the urgency and relevance of collected information.
\end{abstract}

\section{Introduction}

In this paper we present the design, implementation, and empirical analysis of WebRACE, a distributed crawler, filtering processor and object cache. WebRACE is part of a more generic system, called $e R A C E$ (extensible Retrieval, Annotation and Caching Engine), which is a distributed middleware infrastructure that enables the development and deployment of content-delivery and mobile services on Internet [13]. eRACE collects information from heterogeneous

\footnotetext{
* This work was supported in part by the Research Promotion Foundation of Cyprus under grant PENEK-No 23/2000.
} 
Internet sources according to pre-registered, XML-encoded user and service profiles. These profiles drive the collection of information and determine the relevance and the urgency of collected information. eRACE offers a functionality that goes beyond the capabilities of traditional Web servers and proxies, providing support for intelligent personalization, customization and transcoding of content, to match the interests and priorities of individual end-users connected through fixed and mobile terminals. Its goal is to enable the development of new services and the easy re-targeting of existing services to new terminal devices.

WebRACE is the Web-specific proxy of eRACE. It crawls the Web to retrieve documents according to user profiles. The system subsequently caches and processes retrieved documents. Processing is guided by pre-defined user queries and consists of keywords-searches, title-extraction, summarizing, classification based on relevance with respect to user-queries, estimation of priority, urgency, etc. WebRACE processing results are encoded in a WebRACE-XML grammar and fed into a dissemination server, which is designed to select dynamically among a suite of available choices for information dissemination, such as "push" vs. "pull," the formatting and transcoding of data (HTML, WML, XML), the connection modality (wireless vs. wire-based), the communication protocol employed (HTTP, GSM/WAP, SMS), etc.

In the following sections we describe our design effort, and implementation experience with using Java to develop the high-performance Crawler, Annotation Engine and Object Cache of WebRACE. We also describe a number of techniques employed to achieve high-performance, such as distributed design to enable the execution of crawler modules to different machines, support for multithreading, caching of crawling state, customized memory management, employment of persistent data structures with disk-caching support, optimizations of the Java core libraries for TCP/IP and HTTP communication, etc. Finally, we provide performance measurements from typical executions of WebRACE.

The remaining of the paper is organized as follows: Section 2 presents an overview of the WebRACE system architecture and the challenges addressed in our work. Sections 3 and 4 describe the design and implementation of a Crawler and Object Cache, used to retrieve and store content from the Web. Section 5 presents the Filtering Processor that analyzes the collected information, according to user-profiles. Finally, Section 7 summarizes our conclusions.

\section{WebRACE Design and Implementation Challenges}

WebRACE is comprised of two basic components, the Mini-crawler and the Annotation Engine, which operate independently and asynchronously (see Figure 1). Both components can be distributed to different computing nodes, execute in different Java heap spaces, and communicate through a permanent socket link; through this socket, the Mini-crawler notifies the Annotation Engine every time it fetches and caches a new page in the Object Cache. The Annotation Engine can then process the fetched page asynchronously, according to pre-registered user profiles or other criteria. 
In the development of WebRACE we address a number of challenges: First, is the design and implementation of a user-driven crawler. Typical crawlers employed by major search engines such as Google [3], start their crawls from a carefully chosen fixed set of "seed" URL's. In contrast, the Mini-crawler of WebRACE receives continuously crawling directives which emanate from a queue of standing eRACE requests (see Figure 1). These requests change with shifting eRACE-user interests, updates in the base of registered users, changes in the set of monitored resources, etc.

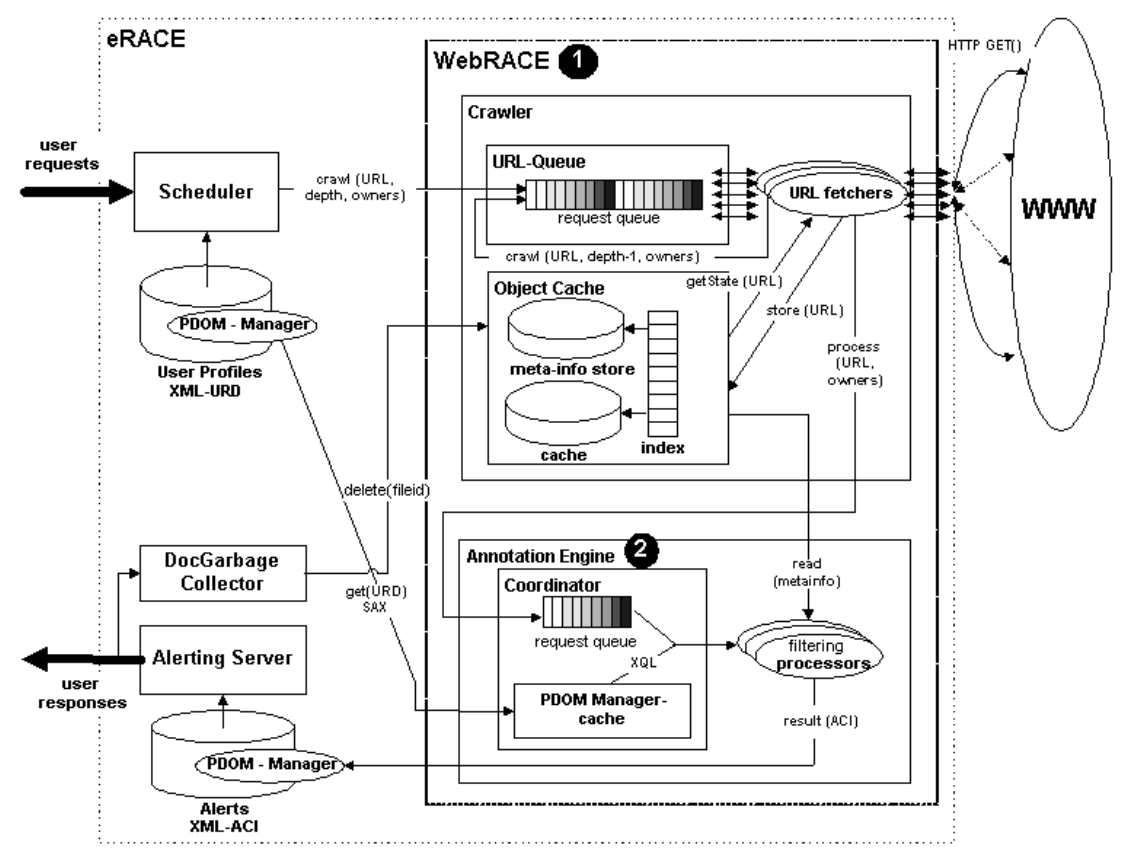

Fig. 1. WebRACE System Architecture.

Second, is the design of a crawler that monitors Web-sites exhibiting frequent updates of their content. WebRACE should follow and capture these updates so that interested users are notified by eRACE accordingly. Consequently, WebRACE is expected to crawl and index parts of the Web under short-term time constraints and maintain multiple versions of the same Web-page in its store, until all interested users receive the corresponding alerts.

Similarly to personal and site-specific crawlers like SPHINX [16] and NetAttache Pro [11], WebRACE is customized and targets specific Web-sites. These features, however, must be sustained in the presence of a large and increasing user base, with varying interests and different service-level requirements. In this context, WebRACE must be scalable, sustaining high-performance and short turn-around times when serving many users and crawling a large portion of 
the Web. To this end, it should avoid duplication of effort and combine similar requests when serving similar user profiles. Furthermore, it should provide built-in support for QoS policies involving multiple service-levels and servicelevel guarantees. Consequently, the scheduling and performance requirements of WebRACE crawling and filtering face very different constraints than systems like Google [3], Mercator [9], SPHINX [16] or NetAttache Pro [11].

Finally, WebRACE is implemented entirely in Java. Its implementation consists of approximately 5500 lines of code, 2649 of which correspond to the Minicrawler implementation, 1184 to the Annotation Engine, 367 to the SafeQueue data structure, and 1300 to common I/O libraries. Java was chosen for a variety of reasons. Its object-oriented design enhances the software development process, supports rapid prototyping and enables the re-use and easy integration of existing components. Java class libraries provide support for key features of WebRACE: platform independence, multithreading, network programming, highlevel programming of distributed applications, string processing, code mobility, compression, etc. Other Java features, such as automatic garbage collection, persistence and exception handling, are crucial in making our system more tolerant to run-time faults.

The choice of Java, however, comes with a certain risk-factor that arises from known performance problems of this programming language and its runtime environment. Notably, performance and robustness are issues of critical importance for a system like WebRACE, which is expected to function as a server, to run continuously and to sustain high-loads at short periods of time. In our experiments, we found the performance of Java SDK 1.3 satisfactory when used in combination with the Java HotSpot Server VM [15,14]. Furthermore, the Garbage Collector, which seemed to be a problem with earlier Java versions, has a substantially improved performance and effectiveness under Java v.1.3.

Numerous experiments with earlier versions of WebRACE, however, showed that memory management cannot rely entirely on Java's garbage collection. During long crawls, memory allocation increased with crawl size and duration, leading to over-allocation of heap space, heap-space overflow exceptions, and system crashes. Extensive performance and memory debugging with the OptimizeIt profiler [20] identified a number of Java core classes that allocated new objects excessively and caused heap-space overflows and performance degradation. Consequently, we had to develop our own data-structures that use a bounded amount of heap-space regardless of the crawl size, and maintain part of their data on disk. Furthermore, we re-wrote some of the mission-critical Java classes, streamlining very frequent operations. More details are given in the sections that follow.

\section{The Mini-crawler of WebRACE}

A crawler is a program that traverses the hypertext structure of the Web automatically, starting from an initial hyper-document and recursively retrieving all documents accessible from that document. Web crawlers are also referred to as robots, wanderers, or spiders. Typically, a crawler executes a basic algorithm 
that takes a list of "seed" URL's as its input, and repeatedly executes the following steps [1]: It initializes the crawling engine with the list of seed URL's and pops a URL out of the URL list. Then, it determines the IP address of the chosen URL's host name, opens a socket connection to the corresponding server, asks for the particular document, parses the HTTP response header and decides if this particular document should be downloaded. If this is so, the crawler downloads the corresponding document and extracts the links contained in it; otherwise, it proceeds to the next URL. The crawler ensures that each extracted link corresponds to a valid and absolute URL, invoking a URL-normalizer to "de-relativize" it, if necessary. Then, the normalized URL is appended to the list of URL's scheduled for download, provided this URL has not been fetched earlier.

In contrast to typical crawlers [16,9], WebRACE refreshes frequently its URL-seed list from requests posted by the eRACE Request Scheduler. These requests have the following format:

[Link, ParentLink, Depth, \{owners $\}]$

Link is the URL address of the Web resource sought, ParentLink is the URL of the page that contained Link, Depth defines how deep the crawler should "dig" starting from the page defined by Link, and \{owners $\}$ contains the list of eRACE users whose profile designates an interest for the pages that will be downloaded.

Making the Mini-crawler configurable through these configuration files renders it adaptable to specific crawl tasks and benchmarks. The crawling algorithm described in the previous section requires a number of components, which are listed and described in detail below:

- The URLQueue for storing links that remain to be downloaded.

- The URLFetcher that uses HTTP to download documents from the Web. The URLFetcher contains also a URL extractor and normalizer that extracts links from a document and ensures that the extracted links are valid and absolute URL's.

- The Object Cache, which stores and indexes downloaded documents, and ensures that no duplicate documents are maintained in cache. The Object Cache, however, can maintain multiple versions of the same URL, if its contents have changed with time.

The Mini-crawler is configurable through three files: a) / conf / webrace . conf, which contains general settings of the engine, such as the crawling start page, the depth of crawling, intervals between system-state save, the size of key datastructures maintained in main memory, etc.; b) /conf/mime.types, which controls what Internet media types should be gathered by the crawler;

c) / conf/ignore.types, which controls what file extensions should be blocked by the engine; URL resources with a suffix listed in ignore.types will not be downloaded regardless of the actual mime-type of that file's content.

\subsection{The URLQueue}

The URLQueue is an implementation of our onw SafeQueue class. We designed and implemented SafeQueue to guarantee the efficient and robust operation of 
WebRACE and to overcome problems of the java.util.LinkList component of Java [6]. We implemented SafeQueue as a circular array of QueueNode objects with its own memory-management mechanism, which enables the re-use of objects and minimizes garbage-collection overhead. Moreover, SafeQueue incorporates support for persistence, overflow control, disk caching, multi-threaded access, and fast indexing to avoid the insertion of duplicate QueueNode entries. More details on the design and implementation of SafeQueue can be found in [24].

URLQueue is a SafeQueue comprised of $U R L Q u e u e N o d e$, i.e., Java objects that capture requests coming from the Request Scheduler of eRACE. During the server's initialization, WebRACE allocates the full size of the URLQueue on the heap. The length of the URLQueue is determined during the server's initialization from WebRACE configuration files. At that time, our program allocates the heap-space required to store all the nodes of the queue. We chose this approach instead of allocating Queue Nodes on demand for memory efficiency and performance. In our experiments, we configured the URLQueue size to two million nodes, i.e., two million URL's. This number corresponds to approximately $27 \mathrm{MB}$ of heap space. A larger URLQueue can be employed, however, at the expense of heap size available for other components of WebRACE.

\subsection{The URLFetcher}

The URLFetcher is a WebRACE module that fetches a document from the Web when provided with a corresponding URL. The URLFetcher is implemented as a simple Java-thread, supporting both HTTP/1.0 and HTTP/1.1. Similarly to crawlers like Mercator [9], WebRACE supports multiple URLFetcher threads running concurrently, grabbing pending requests from the URLQueue, conducting synchronous I/O to download WWW content, and overlapping I/O with computation. In the current version of WebRACE, resource management and thread scheduling is left to Java's runtime system and the underlying operating system. The number of available URLFetcher threads, however, can be configured during the initialization of the WebRACE-server. It should be noted that a very large number of URLFetcher threads can lead to serious performance degradation of our system, due to excessive synchronization and context-switching overhead.

The URLFetcher supports the Robots Exclusion Protocol (REP) that allows Web masters to declare parts of their sites off-limits to crawlers. In addition to supporting the standard Robots Exclusion Protocol, WebRACE supports the exclusion of particular domains and URL's. To implement the exclusion protocol, WebRACE provides a BlockDomain hash table, which contains all domains and URL's that should be blocked. In addition to handling HTTP connections, the URLFetcher processes the documents it downloads from the Web. To this end, it invokes methods of its URLExtractor and normalizer sub-component. The URLExtractor extracts links (URL's) out of a page, disregards URL's pointing to uninteresting resources, normalizes the URL's so that they are valid and absolute and, finally, adds these links to the URLQueue. 


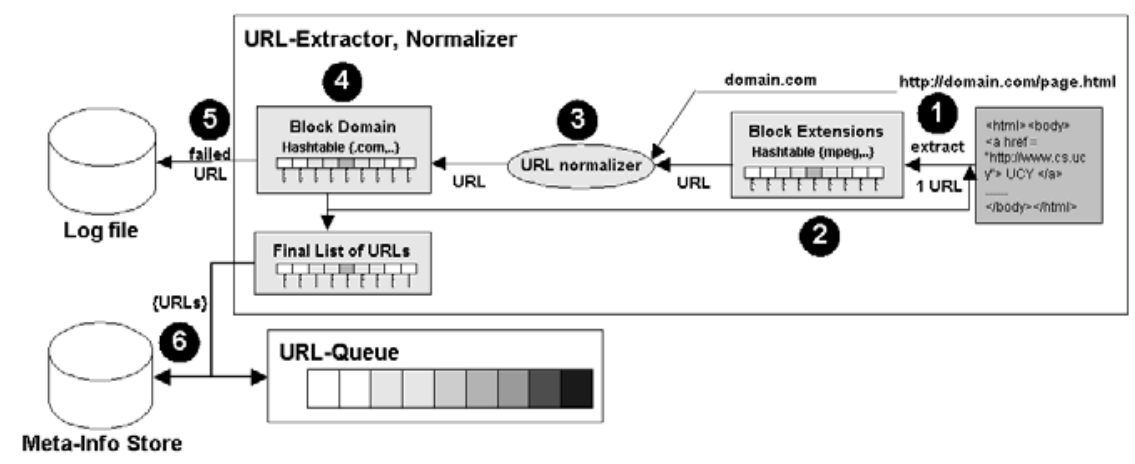

Fig. 2. URL Extractor Architecture.

As shown in Figure 2, the URLExtractor and normalizer works as a 6-step pipe within the URLFetcher. Extraction and normalization of URL's works as follows: in step 1, a fastfind() method identifies candidate URL's in the web-page at hand, removes internal links (starting from "\#"), mailto links ("mailto:"), etc, and extracts the first URL that is candidate for processing. The efficient implementation of fastfind is challenging due to the abundance of badly formed HTML code on the Web. As an alternative solution we could reuse components such as Tidy [18] or its Java port, JTidy [12], to transform the downloaded Web page into well-formed HTML, and then extract all links using a generic XML parser. This solution proved to be too slow, in contrast to our fastfind() method which extracts links from a $70 \mathrm{~KB}$ web page in approximately $80 \mathrm{~ms}$.

In step 2, a Proactive Link Filtering (PLF) method is invoked to disregard links to resources that are of no interest to the particular crawl. PLF uses the /conf/ignore.types configuration file of WebRACE to determine the file extensions that should be blocked during the URL extraction phase. Deciding if a link should be dropped takes less than $1 \mathrm{~ms}$ and saves WebRACE of the unnecessary effort to normalize a URL, add it to the URLQueue, and open an HTTP connection, just to see that this document has a media type that is not collected by the crawler.

Step 3 deals with the normalization of the URL at hand. To this end, we wrote a URL-normalizer method, which alters links that do not comply to the scheme-specific syntax of HTTP URL's, as defined in the HTTP RFCs. The URL-normalizer applies a set of heuristic corrections, which give on the average a $95 \%$ of valid and normalized URL's. If a link cannot be normalized, it is logged for debugging purposes. The URL-normalizer has been tested succesfully on a test case of 150 problematic URL strings which did not conform to the scheme-specific syntax of HTTP URL's. We are continously upgrading the URLnormalizer as new problematic HTTP URL's appear in our log files.

For each Web page processed, the URL-normalizer makes extensive use of the java.net. URL library while checking the syntactic validity of the normalized 


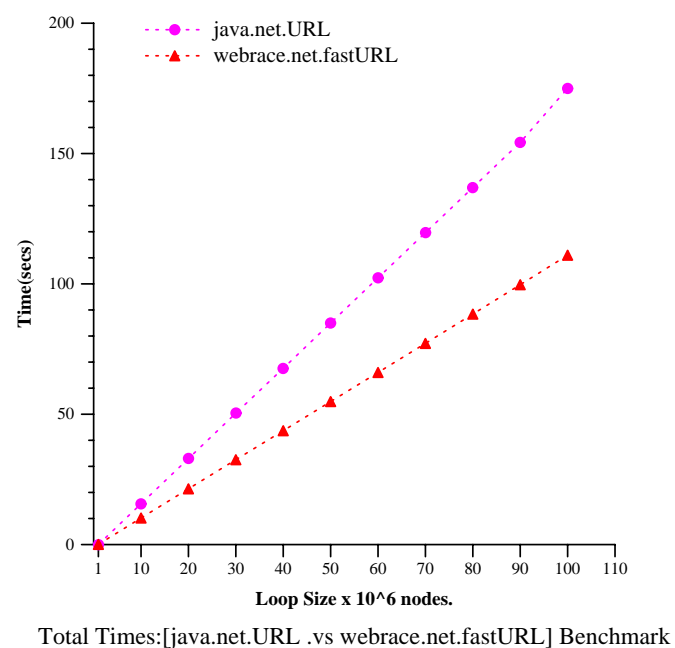

Fig. 3. webrace.net.URL Performance.

URL. This library, however, creates numerous objects that cannot be reused, resulting to excessive heap-memory consumption, an increased activity of the garbage collector, and significant performance degradation. To cope with these problems we implemented webrace. net.fastURL, a streamlined URL class that enables the reuse of URL objects via its reparse (url) method. reparse (url) allows the webrace.net.fastURL() class to disregard previously assigned string values of its private elements host,protocol, port and file, and replace them by new values that need to be validated for conformance to the syntax of HTTP URLs.

This optimization achieves twofold and threefold improvements of the normalization performance under Solaris and Windows NT respectively. Figure 3, presents the results of a java.net.URL vs. webrace.net.fastURL performance benchmark. In this benchmark, we evaluated webrace. net.URL by instantiating up to $10^{8}$ new URL objects. The benchmark ran on a Sun Enterprise E250 Server with 2 UltraSPARC-II processors at $400 \mathrm{M} \mathrm{Hz}$, with $512 \mathrm{MB}$ memory, running the Solaris 5.7 operating system. The URL-normalizer took on the average $200 \mathrm{~ms}$ for 100 URL's.

Step 4 filters out links that belong to domains that are blocked or excluded by the Robot Exclusion Protocols. Steps 1 through 4 are executed repeatedly until all links of the document at hand have been processed. Step 5 logs the URL's that failed the normalization process for debugging purposes. Finally, at step 6 , all extracted and normalized URL's are collectively added to the URL-Queue, dropping all duplicate URL's and URL's that have been visited by the crawler already. The list of normalized URL's is also stored in the Meta-Info Store, so that during re-crawls the Mini-Crawler can avoid URL-extraction of unmodified pages. 


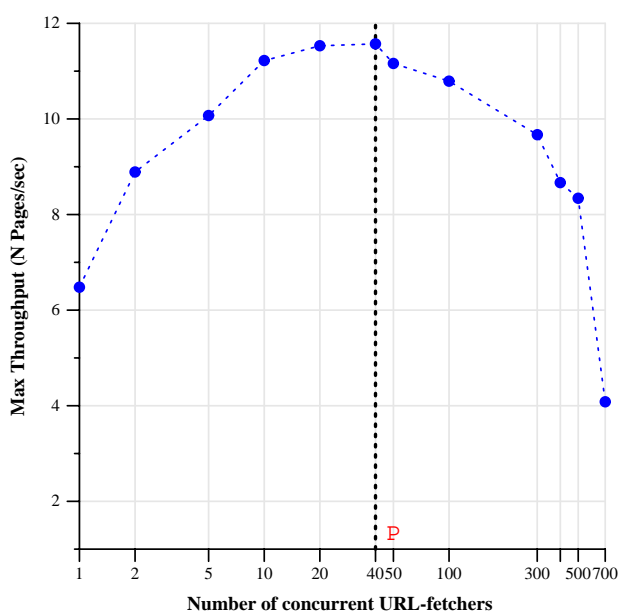

Number of Concurrent URL-fetchers executing in WebRACE (normal-log scale)

Fig. 4. URL-fetcher throughput degradation.

The URL extraction and normalization pipe requires an average of $300 \mathrm{~ms}$ to extract the links from a $70 \mathrm{~KB}$ HTML page and to normalize them appropriately, when executed on our Sun Enterprise E250 Server. To evaluate the overall performance of the URLFetcher, we ran a number of experiments, launching many concurrent fetchers that try to establish TCP connections and fetch documents from Web servers located on our 10/100Mbits LAN. Each URLFetcher pre-allocates all of its required resources before the benchmark start-up. The benchmarks ran on a $360 \mathrm{MHz}$ UltraSPARC-IIi, with 128MB RAM and Solaris 5.7. As we can see from Figure 4, the throughput increases with the number of concurrent URLFetchers, until a peak $\mathrm{P}$ is reached. After that point, throughput drops substantially. This crawling process took a very short time (3 minutes with only one thread), which is actually the reason why the peak value $\mathrm{P}$ is 40 . In this case, URLQueue empties very fast, limiting the utilization of URLFetcher's near the benchmark's end. Running the same benchmark for a lengthy crawl we observed that 100 concurrent URLFetcher's achieve optimal crawling throughput.

Since the optimal crawling throughput can only be determined at runtime we have implemented a Performance Monitoring mechanism which maintains various statistics such as the Connection, Processing and I/O delays which allow us to approximate the optimal number of URLfetcher's running in the system. URLfetchers are consequently either dropped, by a pre-specified percentage (drop_pct\%), or increased slowly (increase_pct\%) on intervals were the system seems to sustain the current load. 


\section{The Object Cache}

The Object Cache is the component responsible for managing documents cached in secondary storage. It is used for storing downloaded documents that will be retrieved later for processing, annotation and subsequent dissemination to eRACE users. The Object Cache, moreover, caches the crawling state in order to coalesce similar crawling requests and to accelerate the re-crawling of WWW resources that have not changed since their last crawl.

The Object Cache is comprised of an Index, a Meta-Info Store and an Object Store (see Figure 1). Although the Index resides in main memory at runtime for increased performance, it is serialized to secondary storage on regular intervals. The documents indexed by the Object Cache are stored on disk allowing us in that way to scale to many billion of documents. The Index of the Object Cache is implemented as a java.util. HashTable, which contains URL's that have been fetched and stored in WebRACE. That way, during re-crawls, URLFetcher's can check if a page has been re-fetched, before deciding whether to download its contents from the Web. The Meta-Info Store collects and maintains metainformation for cached documents. Finally, the Object Store is a directory in secondary storage that contains a compressed version of downloaded resources.

\subsection{Meta-Info Store}

The Meta-Info Store maintains a meta-information file for each Web document stored in the Object Cache. Furthermore, a key for each meta-info file is kept with the Index of the Object Cache to allow for fast look-ups. The contents of a meta-info file are encoded in XML and include:

- The URL address of the corresponding document;

- The IP address of its origin Web server;

- The document size in KB;

- The Last-Modified field returned by the HTTP protocol during download;

- The HTTP response header;

- All extracted and normalized links contained in this document;

- Information about the different document versions kept in the Object Cache.

An excerpt from a meta-info file is given in Table 1. Normally, a URLFetcher executes the following algorithm to download a Web page:

1. Retrieve a QueueNode from the URLQueue and extract its URL.

2. Make the HTTP connection, retrieve the URL and analyze the HTTP-header of the response message. If the host server contains the message "200 Ok," proceed to the next step. Otherwise, continue with the next QueueNode.

3. Download the body of the document and store it in main memory.

4. Extract and normalize all links contained in the downloaded document.

5. Compress and save the document in the Object Cache.

6. Save a generated meta-info file in the Meta-Info Store. 


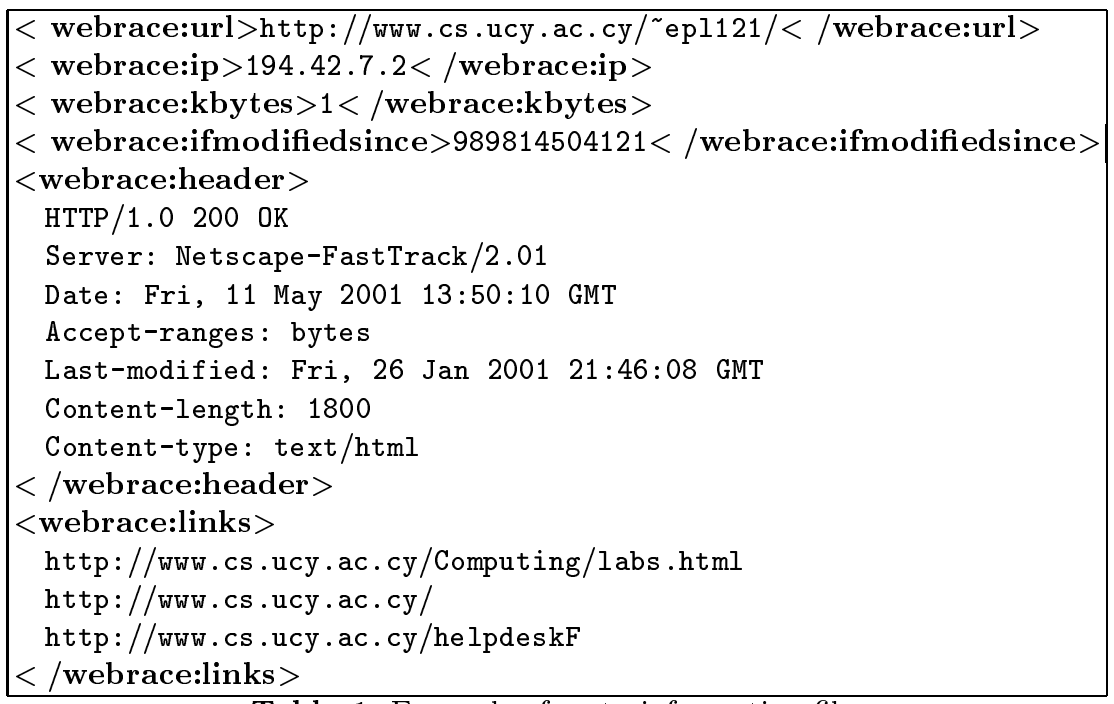

Table 1. Example of meta-information file.

7. Add the key (hashCode) of the fetched URL to the Index of the Object Cache.

8. Notify the Annotation Engine that a new document has been fetched and stored in the Object Cache.

9. Add all extracted URL's to the URLQueue. The URLQueue disregards duplicate URL's in order to avoid leading the crawler into cycles.

To avoid the overhead of the repeated downloading and analysis of documents that have not changed, we alter the above algorithm and use the Meta-Info Store to decide whether to download a document that is already cached in WebRACE. More specifically, we change the second and third steps of the above crawling algorithm as follows:

2. Access the Index of the Object Cache and check if the URL retrieved from the URLQueue corresponds to a document fetched earlier and cached in WebRACE.

3. If the document is not in the Cache, download it and proceed to step 4 . Otherwise:

- Load its meta-info file and extract the HTTP Last-Modified time-stamp assigned by the origin server. Open a socket connection to the origin server and request the document using a conditional HTTP GET command (if-modified-then), with the extracted time-stamp as its parameter.

- If the origin server returns a "304 (not modified)" response and no message-body, terminate the fetching of this particular resource, extract the document links from its meta-info file, and proceed to step 8 . 


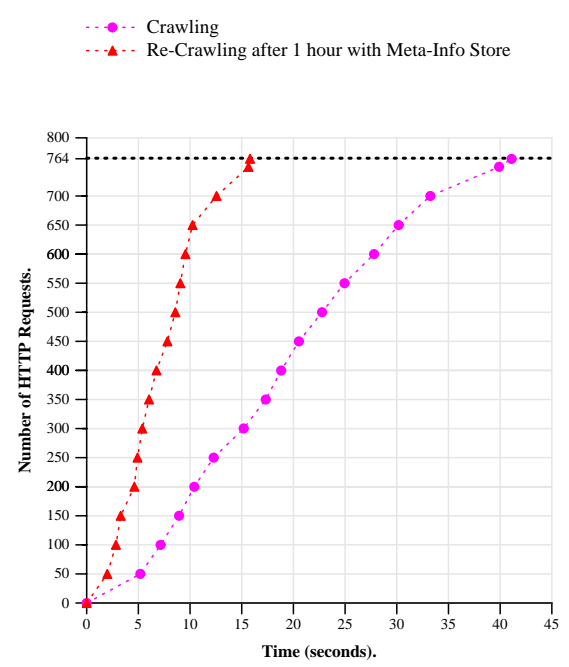

[Crawl vs. Re-crawl, 25 US Universities (2 levels)]

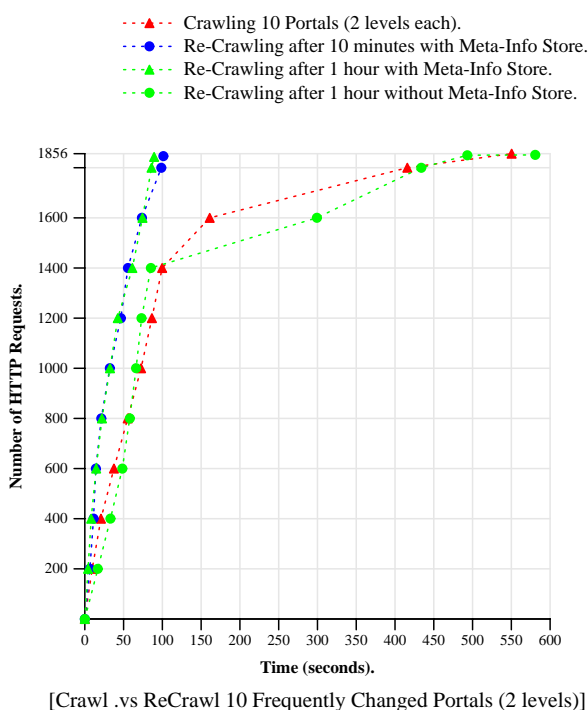

[Crawl .vs ReCrawl 10 Frequently Changed Portals (2 levels)]

Fig. 5. Crawling vs. Re-Crawling in WebRACE in two different settings.

- Otherwise, download the body of the document, store it in main memory and proceed to step 4 .

If a cached document has not been changed during a re-crawl, the URLFetcher proceeds with crawling the document's outgoing links, which are stored in the Meta-Info Store, and which may have changed.

To assess the performance improvement provided by the use of the MetaInfo Store, we conducted an experiment with crawling two classes of Web sites. The first class includes servers that provide content which does not change very frequently (University sites). The second class consists of popular news-sites, search-engine sites and portals (cnn.com, yahoo.com, msn.com, etc.). For these experiments we configured WebRACE to use 150 concurrent URLFetchers and ran it on our Sun Enterprise E250 Server, with the Annotation Processor running concurrently on a Sparc 5.

The diagram of Figure 5a presents the progress of the crawl and re-crawl operations for the first class of sites. The time interval between the crawl and the subsequent re-crawl was one hour; within that hour the crawled documents had not changed at all. The delay observed for the re-crawl operation is attributed to the HTTP "if-modified-since" validation messages and the overhead of the Object Cache. As we can see from this diagram, the employment of the Meta-Info Store results to an almost three-fold improvement in the crawling performance. Moreover, it reduces substantially the network traffic and the Web-servers' load generated because of the crawl.

The diagram of Figure 5b presents our measurements from the crawl and re-crawl operations for the second class of sites. Here, almost $10 \%$ of the 993 


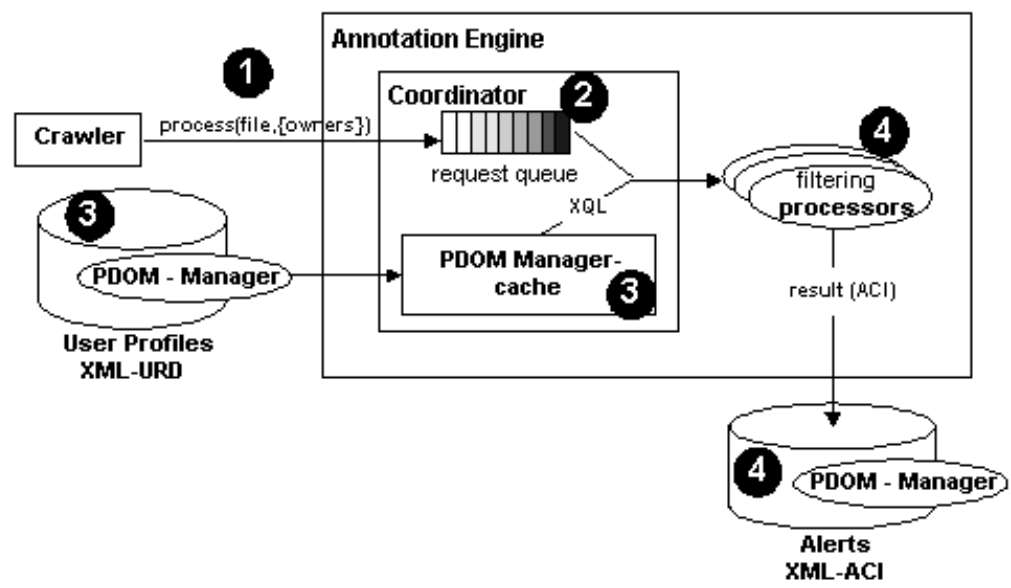

Fig. 6. WebRACE Annotation Engine.

downloaded documents change between subsequent re-crawls. From this diagram we can easily see the performance advantage gained by using the Meta-Info Store to cache crawling meta-information. It should be noted, however, that within the first 100 msecs of all crawl operations, crawling and re-crawling exhibit practically the same performance behavior. This is attributed to the fact that most of the crawled portals reply to our HTTP GET requests with "301 (Moved Permanently)', responses, and re-direct our crawler to other URL's. In these cases, the crawler terminates the connection and schedules immediately a new HTTP GET operation to fetch the requested documents from the re-directed address.

\section{The Annotation Engine (AE)}

The Annotation Engine processes documents that have been downloaded and cached in the Object Cache of WebRACE. Its purpose is to "classify" collected content according to user-interests described in eRACE profiles. The metainformation produced by the processing of the Annotation Engine is stored in WebRACE as annotation linked to the cached content. Pages that are not relevant to any user-profile are dropped from the cache.

Personalized annotation engines are not used in typical Search Engines [1], which employ general-purpose indices instead. To avoid the overhead of incorporating a generic look-up index in WebRACE that will be updated dynamically as resources are downloaded from the Web, we designed the AE so that it processes downloaded pages "on the fly.". Therefore, each time the Annotation Engine receives a " process (file, $\{$ users $\}$ )' ' request through the established socket connection with the Mini-crawler, it inserts the request in the Coordinator, which is a SafeQueue data structure (see Figure 6). Multiple Filtering 
Processors remove requests from the Coordinator and process them according to the Unified Resource Descriptions (URD's) of eRACE users contained in the request. Currently, the annotation engine implements a simple pattern-matching algorithm looking for weighted keywords that are included in the user-profiles, similar to that of [22].

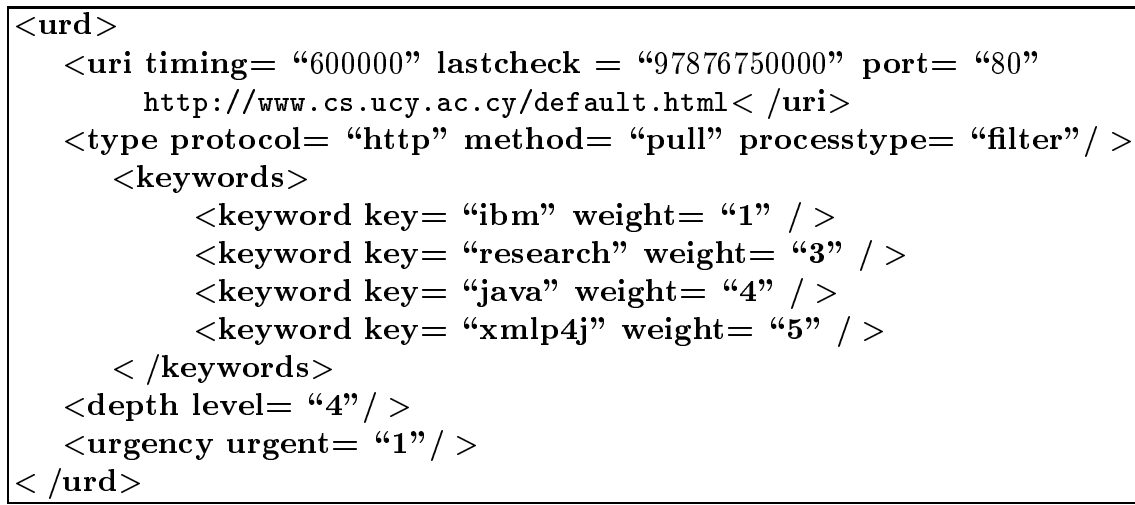

Table 2. A typical URD.

URD is an XML-encoded data structure that encapsulates, within an eRACE user-profile, source information, processing directives and urgency information for Web services monitored by eRACE [23]. A typical URD request is shown in Table 2. URD's are stored in a single XML-encoded document, which is managed by a persistent DOM data manager (PDOM) [10]. The Annotation Engine fetches the necessary URD's from the PDOM data manager issuing XQL queries (eXtensible Query Language) to a GMD-IPSI XQL engine $[10,17]$. The GMD-IPSI XQL engine is a Java-based storage and query application developed by Darmstadt GMD for handling large XML documents. This engine is based on two key mechanisms: a) a persistent implementation of W3C-DOM Document objects [21]; b) a full implementation of the XQL query language. GMD-IPSI provides an efficient and reliable way to handle large XML documents through PDOM, which is a thread-safe and persistent XML-DOM implementation. PDOM supports main-memory caching of XML nodes, enabling fast searches in the DOM tree. A PDOM file is organized in pages, each containing 128 DOM nodes of variable length. When a PDOM node is accessed by a W3CDOM method, its page is loaded into a main memory cache. The default cache size is 100 pages (12800 DOM nodes). Documents are parsed once and stored in Java serialized binary form on secondary storage. The generated document is accessible to DOM operations directly, without re-parsing. The XQL processor is used to query PDOM files.

The output of a filtering process in the Annotation Engine is encoded in XML and called an ACI (Annotated Cache Information) [23]; ACI's are stored 


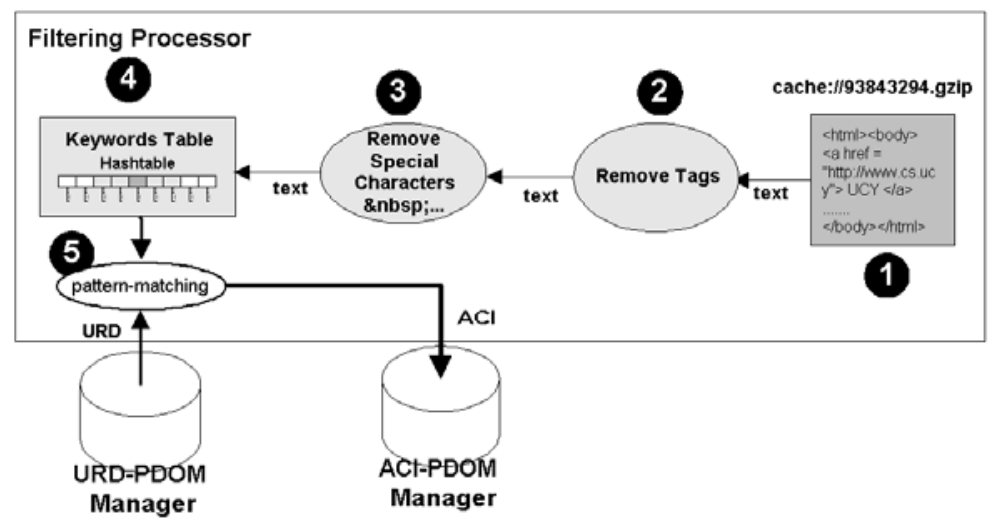

Fig. 7. The Filtering Processor.

in an XML-ACI PDOM database. ACI is an extensible data structure that encapsulates information about the Web source that corresponds to the ACI, the potential user-recipient(s) of the "alert" that will be generated by eRACE's Content Distribution Agents according to the ACI, a pointer to the cached content, a description of the content (format, file size, extension), a classification of this content according to its urgency and/or expiration time, and a classification of the document's relevance with respect to the semantic interests of its potential recipient(s). The XML description of the ACI's is extendible and therefore we can easily include additional information in it without having to change the architecture of WebRACE.

Filtering Processor (FP) is the component responsible for evaluating if a document matches the interests of a particular eRACE-user, and for generating an ACI out of a crawled page (see Figure 7). The Filtering Processor works as a pipe of filters: At step 1, FP loads and decompresses the appropriate file from the Object Cache of WebRACE. At step 2, it removes all links contained in the document and proceeds to step 3, where all special HTML characters are also removed. At step 4, any remaining text is added to a Keyword HashTable. Finally, at step 5, a pattern-matching mechanism loads sequentially all the required URD elements from the URD-PDOM and generates ACI meta-information, which is stored in the ACI-PDOM (step 6). This pipe requires an average of $200 \mathrm{msecs}$ to calculate the $\mathrm{ACI}$ for a $70 \mathrm{~KB}$ Web page, with 3 potential recipients.

In our experiments, we have configured the SafeQueue size of the Annotation Engine to 1000 nodes, which is more than enough, since it is almost every time clear if the AE operates with 10 Filtering Processors and the Mini-crawler with 100 URL-fetchers. We have also observed that the number of pending requests in the $\mathrm{AE}$ SafeQueue has reached a peak of 55 pending requests at a particular run of our system. 


\section{Conclusions}

Although a number of papers have been published on Web crawlers $[16,9,5$, $4,19]$, proxy services and Internet middleware $[2,8]$, the issue of incorporating flexible, scalable and user-driven crawlers in middleware infrastructures remains open. Furthermore, the adoption of Java as the language of choice in the design of Internet middleware and servers raises many doubts, primarily because of performance and scalability questions. There is no question, however, that Web crawlers written in Java will be an important component of such systems, along with modules that process collected content.

In our work, we addressed the challenge of designing and implementing a user-driven, distributed, and scalable crawler and filtering processor, in the context of the eRACE middleware. We described our design and implementation decisions, and various optimizations. Furthermore, we discussed the advantages and disadvantages of using Java to implement the crawler, and presented an evaluation of its performance. To assess WebRACE's performance and robustness we ran numerous experiments and crawls; several of our crawls lasted for days. Our system worked efficiently and with no failures when crawling local Webs in our LAN and University WAN, and the global Internet. Our experiments showed that our implementation is robust and reliable. Furthermore, that caching meta-information about the crawling state can result to significant improvements in crawling performance. Further optimizations will be included in the near future, such as the employment of distributed data structures [7] to make the Mini-crawler itself distributed.

\section{References}

1. A. Arasu, J. Cho, H. Garcia-Molina, A. Paepcke, and S. Raghavan. Searching the Web. ACM Transactions on Internet Technology, 2001. To appear.

2. C. M. Bowman, P. B. Danzig, D. R. Hardy, U. Manber, and M. F. Schwartz. The Harvest Information Discovery and Access System. In Proceedings of the Second International $W W W$ Conference, 1995.

3. S. Brin and L. Page. The Anatomy of a Large-Scale Hypertextual (Web) Search Engine. Computer Networks and ISDN Systems, 30(1-7):107-117, 1998.

4. S. Chakrabarti, M. van den Berg, and B. Dom. Focused Crawling: A New Approach to Topic-Specific Web Resource Discovery. In 8th World Wide Web Conference, Toronto, May 1999.

5. J. Cho, H. Garcia-Molina, and L. Page. Efficient crawling through URL ordering. In Proceedings of the Seventh International WWW Conference, pages 161-172, April 1998.

6. J. Gosling, B. Joy, and G. Steele. The Java Language Specification. AddisonWesley, 1996.

7. S. Gribble, E. Brewer, J. Hellerstein, and D. Culler. Scalable, Distributed Data Structures for Internet Service Construction. In Proceedings of the Fourth Symposium on Operating Systems Design and Implementation (OSDI 2000), 2000.

8. S. Gribble, M. Welsh, R. von Behren, E. Brewer, D. Culler, N. Borisov, S. Czerwinski, R. Gummadi, J. Hill, A. Joseph, R.H. Katz, Z.M. Mao, S. Ross, and B. Zhao. 
The Ninja Architecture for Robust Internet-scale Systems and Services. Computer Networks, 35:473-497, 2001.

9. A. Heydon and M. Najork. Mercator: A Scalable, Extensible Web Crawler. World Wide Web, 2(4):219-229, December 1999.

10. G. Huck, I. Macherius, and P. Fankhauser. PDOM: Lightweight Persistency Support for the Document Object Model. In Proceedings of the 1999 OOPSLA Workshop Java and Databases: Persistence Options. Held on the 14th Annual ACM SIGPLAN Conference on Object-Oriented Programming Systems, Languages, and Applications (OOPSLA '99). ACM, SIGPLAN, November 1999.

11. Tympani Development Inc. NetAttache Pro. http://www.tympani.com/products/NAPro.html, 2000.

12. S. Lempinen. Jtidy. http://lempinen.net/sami/jtidy.

13. M.DIkaiakos and D.Zeinalipour. eRACE Project. http://www.cs.ucy.ac.cy/Projects/eRACE/, 2001.

14. Steve Meloan. The Java HotSpotTM Performance Engine: An In-Depth Look. Technical report, Sun Microsystems, June 1999. http://developer.java.sun.com/developer/technicalArticles/Networking/HotSpot/.

15. Sun Microsystems. The Java HotSpot TM Server VM. http://java.sun.com/products/hotspot/, 1999.

16. R. Miller and K. Bharat. SPHINX: A Framework for Creating Personal, Sitespecific Web Crawlers. In Proceedings of the Seventh International WWW Conference, pages 161-172, April 1998.

17. GMD-IPSI XQL Engine. http://xml.darmstadt.gmd.de/xql/.

18. D. Raggett. Clean up your Web pages with HTML TIDY. http://www.w3.org/People/Raggett/tidy/.

19. S. Raghavan and H. Garcia-Molina. Crawling the Hidden Web. In VLDB 2001: 27th International Conference on Very Large Data Bases, September 2001. To appear.

20. VMGEAR. OptimizeIt!: The Java Ultimate Performance Profiler. http://www.vmgear.com/.

21. W3C. Document Object Model (DOM) Level 1 Specification. W3C Recommendation 1, October 1998. http://www.w3.org/TR/REC-DOM-Level-1/.

22. T. W. Yan and H. Garcia-Molina. SIFT - A Tool for Wide-Area Information Dissemination. In Proceedings of the 1995 USENIX Technical Conference, pages 177-186, 1995.

23. D. Zeinalipour-Yazti. eRACE: an eXtensible Retrieval, Annotation and Caching Engine, June 2000. B.Sc. Thesis. In Greek.

24. D. Zeinalipour-Yazti and M. Dikaiakos. High-Performance Crawling and Filtering in Java. Technical Report TR-01-3, Department of Computer Science, University of Cyprus, June 2001. 